\title{
Modeling and Analysis of Two Node Network Model with Multiple States in Mobile Networks
}

\author{
Patil Shweta \\ Dept. of E\&C \\ VTU Center for PG Studies \\ Bangalore, India
}

\author{
B.N. Manjunatha Reddy \\ Dept. of E\&C \\ Global Academy of Technology \\ Bangalore, India.
}

\begin{abstract}
In this work, the decision probability of the handoff are modeled and simulated for smaller bandwidths. The smaller bandwidth is chosen just for simulation purposes and to demonstrate the applicability of the algorithm. The probability of handover and probability of incorrect decision in the handover is modeled. Two nodes of the network are modeled and the probabilities of four different states of the mobile node are also modeled. The results are presented for two cases with and without the probabilities of four different states of the mobile nodes.
\end{abstract}

Keywords: Wrong Decision Probability, Missing Handovers, Unnecessary handovers

\section{INTRODUCTION}

There are number of networks and technologies available today to meet the market demands and the continuous changing needs of the markets. The technologies and the topologies of the networks are designed and developed based on the requirements per the market. The networks are designed to handle enough bandwidth, signal strength, speed of handling and processing of data, voice and videos. More importantly, the networks should provide the guaranteed security so that the data transmission is safe. This is an essential requirement for the corporates using these networks in their day to day work. Another important factor one should consider when designing these networks is there should be a reliable handover of the mobile nodes when the certain condition like movement of mobile node, bandwidth availability and signal strength are considered as a condition of handover. The challenge is how smoothly one can handover the mobile node seamlessly.

Handover is performed when certain conditions in the network system are met. These conditions have been used to design multiple numbers of algorithms [1]. The conditions are based on the factors like bandwidth availability in the target network versus the present network, signal strength, movement of the mobile node, access delay. For example, when the user of the mobile node moves from one area to another area in a locomotive or an automobile, the handover happens based on the distance between the network node and the mobile node. If the distance of another network with respect to the position of the mobile node is less compared to that of the present one, handover happens. Similarly, the handover can happen if the received signal strength by the mobile node is less than certain threshold [1]. However, it must be built into the algorithm that if a mobile node is handed over based on the signal strength or movement of the mobile node, there should be availability of enough bandwidth in the target network. If bandwidth is not available, then the algorithm should also resolve such conflicts. These kinds of algorithms are already available in the literature [2].

While there is sufficient number of handover algorithms, there is lack metrics or gauge algorithm which measures the performance of the handover algorithms. The performance needs to be measured against certain criteria like number of successful handovers, number of incorrect decisions of handover etc. Hence it is required to develop a common method based on analytical models for better comparison. There are already some algorithms available which can measure the performance of the handover algorithms [3, 4]. The criteria used in these algorithms are based on received signal strength $[3,4]$. There is another algorithm available which is based on the access delay [2]. The disadvantage of these algorithms is lack of robust analytical model in the structure. This gap in the measurement of performance of algorithms necessitated the concept of the Wrong decision probability model [6]. This model measures the number of incorrect decisions made by the handover algorithms irrespective of the kind of decision criteria used for the handover. In other words, the wrong decision probability model measures the performance of the handover algorithm if it is based on bandwidth, signal strength or movement of mobile node.

The performance of all the algorithms mentioned above are evaluated in the virtual environment and the measured performance is only applicable to the environment having conditions made in assumptions while developing the algorithms. The actual performance in real situation may be quite different from the simulated performance. Other algorithms can be found in references [7-20].

In this work, an improvement is made to the wrong decision model developed in ref [6]. Four different states of the mobile node [21] are considered in this work. The mobile node can stay in any of the two network nodes and in any of the four states. Semi Markov models are used to represent the actual scenario and the probabilities are considered to evaluate the performance of the two node network model.

In this work, probability models are used to determine the probabilities of unnecessary handovers, missing handovers and wrong decisions for different decision times and for two different models, namely, baseline and proposed models. Next section present the mathematical models used in the analytical formulations. Section III describes the general algorithms used and section IV explains the simulated results based on MATLAB coding. Finally important conclusions are drawn in section VI. 


\section{ANALYTICAL MODEL}

There are two network nodes in the model, namely, $n 1$ and $n 2(\mathrm{P}$ and $\mathrm{Q})$. Assume that the mobile node is either in network node $\mathrm{P}$ or $\mathrm{Q}$. The mobile node while in network nodes $\mathrm{P}$ or $\mathrm{Q}$ can be in any of the four states. These four states are:

1. Cooperative State

2. Malicious State

3. Selfish State

4. Failed State.

\section{Definitions:}

- $\quad$ In Cooperative state, the mobile nodes are active in route discovery and packet forwarding, but not in DOS attack launching.

- In Malicious State, the mobile nodes are active in route discovery and DOS attack launching.

- In Selfish state, the mobile nodes are active in route discovery, but not in packet forwarding and DOS attack launching.

- In failed State, the mobile nodes are not active in route discovery.

The models proposed by Chi et. al [6] have only two network nodes with just one state, i.e. cooperative state of mobile node.

The two models may be called as

1. Baseline model (Proposed by Chi et.al [6])

2. Improved model (Proposed in this work)

\section{A. Baseline Model:}

Let there are two wireless networks. As mentioned in the baseline model [6], the probability that a mobile node continues to stay in the network node 1 is,

$P_{n 1}=\frac{P_{n 1 / n 2}}{P_{n 1 / n 2}+P_{n 2 / n 1}}$

And, the probability that a mobile node continues to stay in the network node 2 is,

$P_{n 2}=\frac{P_{n 2 / n 1}}{P_{n 1 / n 2}+P_{n 2 / n 1}}$,

Where, $n_{1}$ and $n_{2}$ are network nodes;

$B_{1}$ and $B_{2}$ are maximum available bandwidth for the two networks;

$P_{n j / n i}$ is the probability of mobile node moving from node $n_{i}$ to $n_{j}$

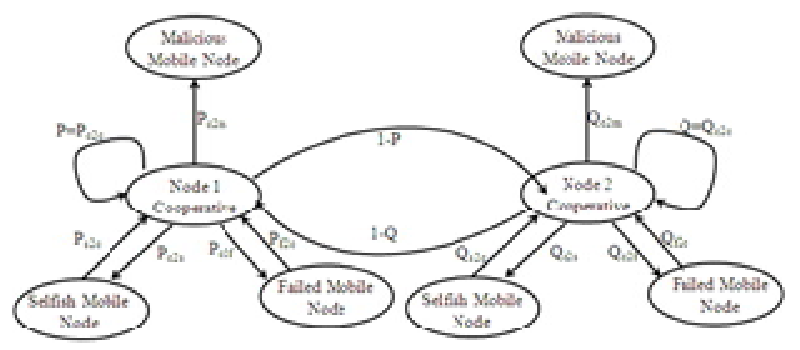

Figure 1: Semi Markov State Two Node Network Model

$P_{n i / n i}$ denotes the probability of mobile node continue to stay in $n_{i}$ after a time interval $D$.

\section{B. Proposed Model:}

In the proposed model as shown in fig.1, the probability that mobile node stays in node 1 is

$P_{c 2 c-1}=\frac{P_{n 1 / n 2}}{P_{n 1 / n 2}+P_{n 2 / n 1}}$

and the probability that a mobile node continues to stay in the network node 1 and in cooperative state is,

$P_{n 1}=P_{c 2 c-1}+P_{f 2 c-1}+P_{s 2 c-1}-P_{c 2 s-1}-P_{c 2 m-1}-P_{c 2 f-1}$

The probability that mobile node stays in node 2 ,

$P_{c 2 c-2}=\frac{P_{n 2 / n 1}}{P_{n 1 / n 2}+P_{n 2 / n 1}}$

and, the probability that a mobile node continues to stay in the network node 2 and in cooperative state is,

$P_{n 2}=P_{c 2 c-2}+P_{f 2 c-2}+P_{s 2 c-2}-P_{c 2 s-2}-P_{c 2 m-2}-P_{c 2 f-2}$

Where, probability of the mobile node moving from failed state to cooperative state is given by,

$P_{f 2 c}=\frac{1}{T_{\text {Recover }}}$

Probability of the mobile node moving from selfish state to cooperative state is given by,

$P_{s 2 c}=\frac{T C_{T h r}}{T C_{\text {Max }}}$

Probability of the mobile node moving from cooperative state to selfish state is given by,

$$
P_{c 2 s}=\frac{1}{T_{\text {Selfish }}}
$$

Probability of the mobile node moving from cooperative state to malicious state is given by,

$P_{c 2 m}=q_{a} \frac{k_{a}}{N} \frac{1}{T_{\text {attack }}}$

Probability of the mobile node moving from cooperative state to failed state is given by, 


$$
P_{c 2 f}=\max \left(\frac{1}{T_{\text {Life }}}, \frac{1}{T_{\text {Residence }}}\right)
$$

More details about these equations and nomenclature can be found in ref [21]. Handover probabilities are given by,

$H P=P_{n 1} P_{n 2 / n 1}+P_{n 2} P_{n 1 / n 2}$

Wrong decision probability can be computed from the unnecessary handover probability (UHP) and missing handover probability (MHP). The exact expressions for the probabilities can be seen in reference 6 . The difference lies only in the computation of the probabilities $P_{n 1}$ and $P_{n 2}$. Wrong decision probability is the summation of UHP and MHP i.e.

$W D P=U H P+M H P$

\section{GENERAL ALGORITHM}

Only the band width is considered as the criteria for the handover. The general algorithm that is used in this simulation is similar to the one used in the reference 6 .

\section{SIMULATION RESULTS}

In this section the simulation results are presented for a maximum bandwidth of the 16 in both the network nodes 1 and 2. MATLAB is used for coding the probability models. The traffic density is varied from 1 to 15 and for six cases of the decision or life time of the mobile node. The decision times of 10 to 15 time units are modeled. The following factors are used in the simulations:

- $\quad$ Life time of the mobile node $=$ Decision time

- $\quad$ Residence time $=$ Decision time/4

- $\quad$ Attack time $=$ Decision time $/ 4$

- $\quad$ Recovery time $=$ Decision time $/ 4$

- $\quad$ Selfish time $=$ Decision time/4

- $\quad$ probability of attack, $q a=0.3$

- $\quad \mathrm{TC}_{\mathrm{thr}} / \mathrm{TC}_{\max }=0.01$

- $\mathrm{ka} / \mathrm{N}=0.01$

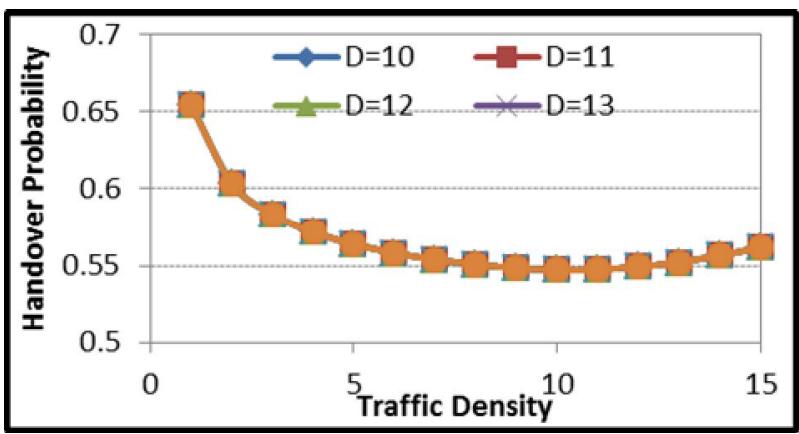

Figure.2: Handover probability versus Traffic
Density for Baseline Model

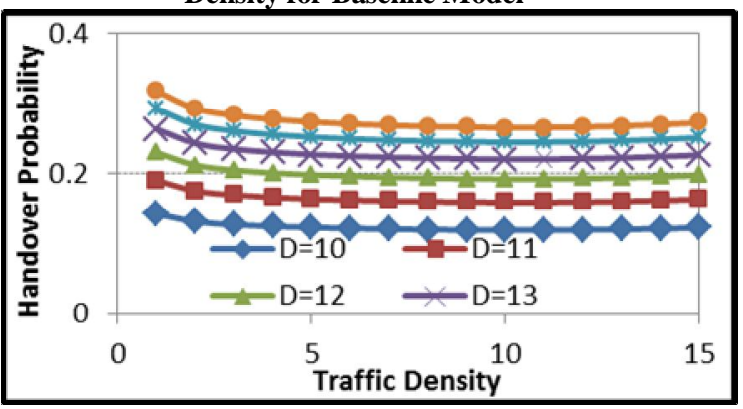

Figure.3: Handover Probability versus Traffic Density for Proposed Model

Fig. 2 shows the handover probability versus traffic density for baseline model for different decision times. It can be noticed that the handover probability is independent of the decision time. This assumption is not a valid assumption as per the baseline model proposed in reference 6 . Because the conditions of the handover can change at the other networks when the decision times are large. For example, the bandwidth availability in the target network may change by the time the mobile node is actually handed over.

Fig. 3 shows the handover probability versus traffic density for proposed model. These probabilities represent the reality as the handover probability varies with respect to the decision time. Also, the the handover probability is very high in the baseline model compared to the proposed model.

Fig. 4 and Fig. 5 show the unnecessary handover probability versus traffic density for baseline model and poposed models respectively. It can be noticed that the unnecessary handover probabilities are less by around $50 \%$ in the proposed models. With the baseline models, the probability models are oversimplified and hence high values of probabilities. The probabilities start to take the reverse profile after $D=10$.

Fig. 6 and Fig. 7 show the missing handover probability versus traffic density for baseline model and proposed models respectively. It can be observed that the missing handover probabilities are less by around $70 \%$ in the proposed models. The results start to take reverse profile after the decision time of 15 time units.

\section{CONCLUSION}

In this work the probability models are developed using four states of the mobile node namely, cooperative, selfish, failed and malicious states. The mobile node can move from any one of the state to other states. What is important in this model is, one the cooperative state is useful for the handover and all the probabilities moving from and into this state is considered for calculating the wrong decision probability. It is observed that the wrong decision probabilities are predicted much accurately by at least $60 \%$ higher than the baseline model proposed in reference 6 . It is also demonstrated that at certain values of the decision time the results start to take the reverse profiles for the factors used in this model. Based on this one can decide on the values of factors for the kind of probabilities that is of interest to the user. These models can be further extended to consider the signal strength, movement of the mobile node, access delays etc. 


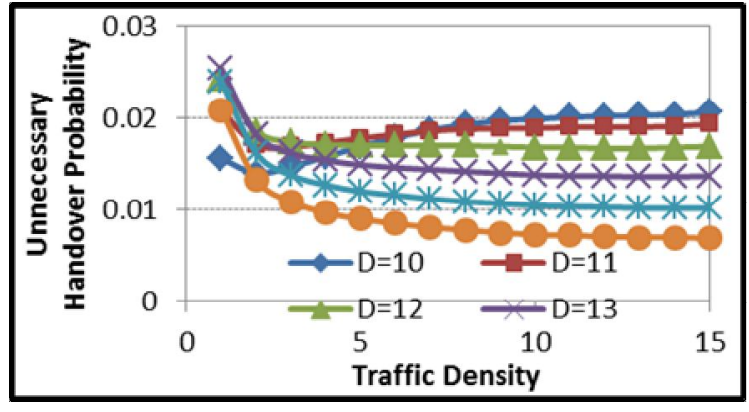

Figure 4: Unnecessary Handover Probability versus Traffic Density for Baseline Model

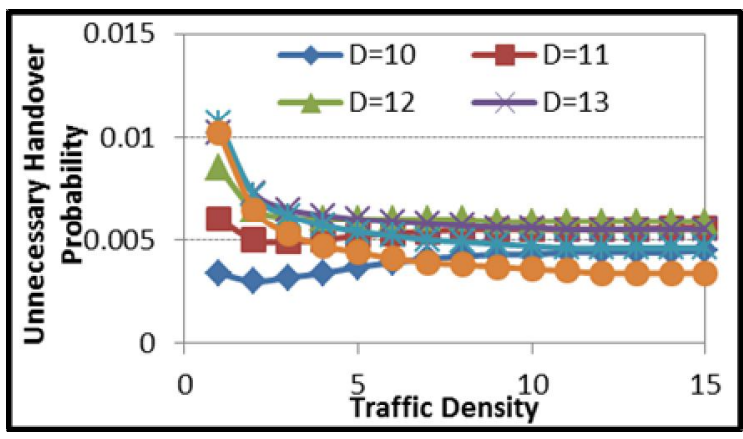

Figure 5: Unnecessary Handover Probability versus Traffic Density for Proposed Model

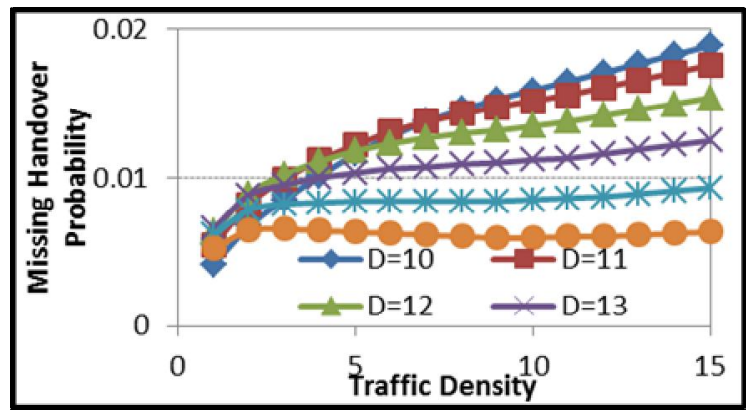

Figure 6: Missing Handover Probability versus Traffic Density for Baseline Model

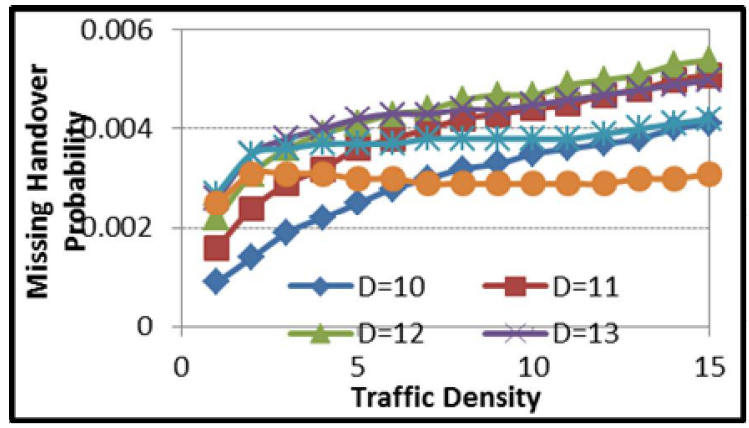

Figure 7: Missing Handover Probability versus Traffic Density for Proposed Model

\section{REFERENCES}

[1]. A. H. Zahran, B. Liang, and A. Saleh, "Signal threshold adaptation for vertical handoff in heterogeneous wireless networks," Mob. Netw. Appl., vol. 11, no. 4, pp. 625640, 2006.

[2]. Chuanxiong Guo, Zihua Guo, Qian Zhang, and Wenwu Zhu, "A Seamless and Proactive End-to-End Mobility Solution for Roaming Across Heterogeneous Wireless Networks", IEEE Journal on Selected Areas in Communications, Vol. 22, No. 5, June 2004, pp.834-848.

[3]. W. Zhao, R. Tafazolli, and B. G. Evans, "Internetwork handover performance analysis in a gsm-satellite integrated mobile communication system," Selected Areas in Communications, IEEE Journal on, vol. 15, no. 8, pp. 1657-1671, 1997.

[4]. M. N. Halgamuge, H. Le Vu, K. Rarnamohanarao, and M. Zukerman, "Signal-based evaluation of handoff algorithms," Communications Letters, IEEE, vol. 9, no. 9, pp. 790-792, 2005.

[5]. H.Sanneck and G.Carle, "A Framework Model for Packet Loss Metrics Based on Loss Runlengths", Proceedings of the SPIE/ACM SIGMM Multimedia Computing and Networking Conference 2000.

[6]. C. Chi, X. Cai, R. Hao and F. Liu "Modeling and Analysis of Handover Algorithms" IEEE GLOBECOM 2007 proceedings.

[7]. Q. Zhang, C. Guo, Z. Guo, and W. Zhu, "Efficient Mobility Management for Vertical Handoff between WWAN and WLAN," IEEE Commun. Mag., November 2003.

[8]. K. Egevang and P. Francis, "The IP network address translator (NAT)," RFC1631.

[9]. Daniel Heyman and Mattlew Sobel. Stochastic Models in Operations Research. McGraw-Hill, 1982.

[10]. G. Pollini, "Trends in handover design," IEEE Commun. Mag., March, 1996.

[11]. K. Pahlavan, P. Krishnamurthy, A. Hatami, M. Ylianttila, J. Makela, R. Pichna, and J. Vallstrom, "Handoff in Hybrid Mobile Data Networks," IEEE Personal Communications, Apr. 2001.

[12]. M. Ylianttila, M. Pande, J. Makela, and P. Mahonen, "Optimization scheme for mobile users performing vertical handoffs between IEEE 802.11 and GPRS/EDGE networks," in Proc. IEEE Globecom'01.

[13]. Xiang-Yang Li, Peng-Jun Wan, Yu Wang, and Chih-Wei Yi. Fault Tolerant Deployment and Topology Control in Wireless Networks. In Proc. of ACM MobiHoc '03, pages 117-128, Jan. 2003.

[14]. EURESCOM Project P1013-FIT-MIP, "First steps towards UMTS: Mobile IP services, a European testbed", http://www.eurescom.de/public/projects/P1000series/p1013/default.asp.

[15]. J. McNair, I. Akyildiz, and M. Bender, "An inter-system handoff technique for the IMT-2000 system," in Proc. IEEE infocom 2000.

[16]. C. Perkins, Editor, "IP mobility support for IPv4," RFC 3344, http://www.ietf.org/rfc/rfc3344.txt

[17]. C. Perkins, et al., "Mobility support in IPv6," IETF draft, http://www.ietf.org/internet-drafts/draftietfmobileip- ipv6-24.txt, work in progress, June, 2003.

[18]. Hao Yang, Haiyun Luo, Fan Ye, Songwu Lu, and Lixia Zhang. Security in Mobile Ad Hoc Networks: Challenges and Solutions. IEEE Wireless Communications, pages 38 - 47, Feb 2004.

[19]. A. Valko, "Cellular IP: A New Approach to Internet Host Mobility," Computer and Communication Review, vol. 29, no.1, pp. 50-65, January 1999. 